\title{
Contagious epididymitis due to Brucella ovis: relationship between sexual function, serology and bacterial shedding in semen
}

Nicole Picard-Hagen ${ }^{1}$, Xavier Berthelot ${ }^{2}$, Jean Luc Champion ${ }^{3}$, Laure Eon ${ }^{4}$, Faouzi Lyazrhi ${ }^{5}$, Maxime Marois $^{3}$, Marceline Peglion ${ }^{6}$, Aude Schuster ${ }^{2}$, Christel Trouche ${ }^{2}$ and Bruno Garin-Bastuji ${ }^{7} 8^{*}$

\begin{abstract}
Background: Contagious Epididymitis (CE) due to Brucella ovis (B. ovis) is a contagious disease that impairs rams' fertility due to epididymis, testicle and accessory sexual gland alterations. An increased incidence of CE has been observed in South Eastern France ("PACA" region) since the Rev.1 vaccination against B. melitensis has been stopped in 2008. The objective of this study was to evaluate the relationship between the infection by B. ovis and the sexual function of rams.

Two-hundred eighteen sexually-mature rams, from 11 seropositive flocks, were submitted to a clinical examination of the genital tract, a semen collection by electro-ejaculation for spermogram and culture, and a serological examination for anti-B. ovis antibodies by complement fixation test (CFT) and indirect ELISA (I-ELISA). The relationships between clinical, seminal, bacteriological and serological parameters were studied using the Fisher exact test and a logistic regression model (binomial logit).

Results: $B$. ovis shedding in semen was significantly associated with seropositivity (CFT and I-ELISA; $p<0.001$ and 0.01 respectively), genital tract alterations $(p<0.05)$ and poor semen quality $(p<0.001)$. Seropositive rams presented significantly more genital tract alterations $(p<0.001)$ and a poor seminal score $(p<0.001)$ than seronegative rams.

Conclusions: Since semen culture is not routinely feasible in field conditions, a control plan of CE should be based, where Rev.1 vaccination is not possible, on both systematic clinical and serological examination of rams, followed by the culling of seropositive and/or genital tract alterations carrier rams.
\end{abstract}

Keywords: Brucella ovis, Semen, Sheep, Genital disease

\section{Background}

Contagious Epididymitis (CE) due to Brucella ovis (B. ovis) is a contagious disease of worldwide importance that impairs fertility in rams due to epididymis, testicle and accessory sexual gland alterations. More rarely, abortions in ewes and increased perinatal mortality rates may be observed as well $[1,2]$. Transmission occurs via passive venereal infection or direct contact [2]. The

\footnotetext{
* Correspondence: bruno.garin-bastuji@anses.fr

${ }^{7}$ Paris-Est University/French Agency for Food, Environmental and Occupational Health and Safety (ANSES), EU/OIE/FAO Brucellosis Reference Laboratory, 94701 Maisons-Alfort, France

${ }^{8}$ Present Address: ANSES, European \& International Affairs Department, 14 rue Pierre et Marie Curie, F-94701 Maisons-Alfort, Cedex, France

Full list of author information is available at the end of the article
}

disease can result in significant economic losses in infected flocks where no control programs are in place, due to reproductive failure, culling of breeding animals as well as ban on trade. In the European Union, no compulsory surveillance of the disease is currently in place in flocks, while neither eradication programme nor compensation scheme for culling animals in infected flocks is foreseen. Nevertheless, in order to avoid the contamination of non-infected areas or flocks through international trade, rams have to undergo serological pre-movement tests [2]. On farms, diagnosis mainly relies on a clinical detection and a serological test when the palpation of testicles reveals lesions or when there is significant infertility in the flock $[1,3]$. 
An increased incidence of $\mathrm{CE}$ has been observed in South Eastern France ("PACA" region) since the Rev.1 vaccination against $B$. melitensis has been stopped in 2008 due to eradication of brucellosis in domestic ruminants 5 years before in the whole country [3]. In 2011, the flock prevalence rate (at least 1 seropositive ram per flock) ranged from $5.5 \%$ to more than $50 \%$ according to the area.

The aim of this study was to examine the relationship between B. ovis infection, evaluated through clinical, serological and bacteriological findings, and the sexual function, in rams originated from infected flocks of this region where CE appears enzootic.

\section{Methods}

\section{Animals}

Two-hundred eighteen sexually-mature rams from 11 seropositive flocks from the PACA region of SouthEastern France were included in this study in September 2012. Both seropositive and seronegative animals (based on results of tests performed in former spring) were selected by voluntary breeders in a balanced manner, as much as possible. Breeds of rams were as follows: Merinos $(n=69)$, Préalpes du Sud $(n=30)$, meat breeds (Ile de France and Texel; $n=91$ ); Merinos crossbreeds $(n=15)$, others $(n=13)$.

\section{Breeding soundness assessment}

For each animal, the following parameters were recorded: age, body condition score (BCS), scrotal circumference $(\mathrm{SC}$, in $\mathrm{cm})$, as well as clinical alterations of the genital tract investigated by palpation and, in suspicious cases, by ultrasonography [4].

The Body Condition Score (BCS) was assessed according to the technique described by Russel [5]. Briefly, the BCS was noted from 0 (extremely emaciated) to 5 (obese) by the palpation of the lumbar region (spinous and transverse processes, muscles and fatty tissues), a
BCS of 3 to 4 being considered as optimal during the breeding season [6].

Testicle alterations included asymmetry, indurations, degeneration and/or atrophy, while epididymis alterations included epididymitis, indurations, nodules, hypertrophy and/or cysts.

In order to classify the rams for their breeding potential, a clinical score and a seminal score based upon a point-score system adapted from Ley et al. [7] were calculated for each ram. The clinical score took into account the age, BCS, SC and the presence/absence of clinical signs, as shown in Table 1.

\section{Animals' handling and sample collection}

All samples were collected in the frame of the B. ovis infection control programme implemented by the Fédération Régionale des Groupements de Défense Sanitaire Provence Alpes Côte d'Azur (FRGDS PACA) as approved by its advisory board and in accordance with the EU (in particular Directive 2010/63/EU) and French regulations regarding ethics and best practices of veterinary care.

Animals' handling and sample collection were performed by trained technicians of the FRGDS PACA, veterinary surgeons and graduating veterinary students (under the supervision of 2 professors), with the help of the stockbreeders who had brought their own rams and given their informed consent. Blood samples were collected from each ram for serological examinations. Semen was collected by electroejaculation (Electrojac, Ideal $^{\oplus}$ Instruments, MI, USA) for further laboratory examinations. To preserve the welfare of the ram, the stimulation was discontinued if signs of stress or physical discomfort were detected.

\section{Laboratory examinations}

Each blood serum was subjected to both complement fixation test (CFT) and indirect ELISA (I-ELISA; IDEXX

Table 1 Clinical score calculation and rams classification

\begin{tabular}{|c|c|c|c|}
\hline & \multicolumn{3}{|l|}{ Note } \\
\hline & 1 & 4 & 7 \\
\hline Age (years) & $\geq 5$ & 1 & $2-4$ \\
\hline $\mathrm{BCS}^{*}$ & $<2$ & $>3$ & $2-3$ \\
\hline $\mathrm{SC}(\mathrm{cm})^{* *}$ & $<33$ & $\geq 33-<39$ & $\geq 39$ \\
\hline \multirow[t]{3}{*}{ Epididymis/testicle alterations*** } & Presence $($ note $=0$ ) & $N / A^{* * * *}$ & Absence (note $=7$ ) \\
\hline & \multicolumn{3}{|l|}{ Clinical score } \\
\hline & $3-12$ & $13-21$ & $22-28$ \\
\hline Rams classification & Poor & Fair & Good \\
\hline
\end{tabular}

*BCS: body condition score (0-5) 
Table 2 Seminal score calculation and rams classification

\begin{tabular}{llll}
\hline & Note & \\
& 1 & 4 & 7 \\
\hline Total number of spermatozoa/ejaculate $\left(\times 10^{9}\right)$ & $=<1$ & $>1-\leq 2$ & $>2$ \\
Individual motility (\%) & $=<30$ & $>30-\leq 70$ & $>70$ \\
Normal spermatozoa (\%) & $=<50$ & $>50-\leq 70$ & $>70$ \\
& Seminal score & \\
& $3-9$ & $12-15$ & $18-21$ \\
Rams classification & Poor & Fair & Good \\
\hline
\end{tabular}

Brucella ovis Ab Test; Idexx Montpellier, France) as described by Praud et al. [8]. The CF antigen (Anses, Maisons-Alfort, France) was standardised against the International Standard anti-Brucella ovis Serum (ISaBoS) and the positivity threshold was 50 international CFT units (ICFTU)/mL according to OIE requirements [2]. IELISA was standardized against ISaBoS with the $1 / 64$ pre-dilution of the ISaBoS made up in a negative pool of sera as the minimum detection requirement and the $1 /$ 256 dilution at which the standard must be classified as negative. All tests were performed by the same two technicians at the Anses Brucellosis Reference Laboratory (Anses, Maisons-Alfort, France). In a previous work, Praud et al. [8] suggested that depending on the aim of the test performance (screening, diagnosis confirmation, export control) and disease situation (free, enzootic), different cut-offs could be used (30\%, $45 \%$ or $60 \%)$ but found that the $45 \%$ cut-off gave the best concordance with CFT. In this study, I-ELISA results were therefore interpreted either with a unique cut-off of $45 \%$ (negative or positive) or with a double-cut-off of $30 \%$ and $60 \%$ respectively (negative, doubtful or positive).

For the semen examination, the following characteristics were recorded: volume $(\mathrm{mL})$, gross and progressive motility, sperm morphology and abnormalities after eosin-nigrosin staining; sperm concentration (spermatozoa per $\mathrm{mL}$ ) was measured by spectrophotometry (Accuread, IMV technologies, L'Aigle, France). The seminal score was based on the number of spermatozoa in the ejaculate, the individual motility and the percentage of normal spermatozoa, as shown in Table 2.

Semen cultures for $B$. ovis were performed on samples stored frozen, on non-selective $5 \%$ equine-serum added blood-agar base $\mathrm{N}^{\circ} 2$ (Oxoid, France) in an atmosphere of 5-10\% $\mathrm{CO}_{2}$, at the ANSES Brucellosis Reference Laboratory [2].

\section{Statistical methods}

Statistical analysis was performed using the Fisher exact test and a logistic regression model (binomial logit) with the $\mathrm{R}$ software (http://www.R-project.org). A threshold of $5 \%(p<0.05)$ was considered as significant.

Kappa statistics were also used to assess the agreement between different diagnostic methods.

\section{Results}

\section{Clinical and semen laboratory examinations}

The clinical and seminal characteristics of the 218 rams are presented in Table 3. In 73 rams, clinical alterations were observed by palpation, mainly on epididymes ( $n=$ 60 ; head and tail hypertrophy, indurations and nodules) and testicles $(n=13$; asymmetry, indurations, degeneration and atrophy); 17 alterations were bilateral, 56 unilateral. 145 rams had no macroscopic alteration of the genital tract. Respectively $26 \%$ and $37 \%$ of rams had a good clinical or seminal score.

There was a significant relationship between seminal and clinical score $(p=0.0084)$, mainly explained by the presence (or not) of clinical alterations but not by the $\mathrm{SC}$ or age.

Table 3 Clinical and seminal characteristics of the 218 rams

\begin{tabular}{|c|c|c|c|c|c|}
\hline Parameter & Mean & SD & Median & Min. & Max. \\
\hline Age (years) & 3.3 & 1.7 & 3 & 1 & 9 \\
\hline $\mathrm{BCS}^{*}$ & 2.4 & 0.7 & 2.3 & 1.5 & 3.8 \\
\hline $\mathrm{SC}(\mathrm{cm})^{* *}$ & 36.4 & 3.6 & 34 & 26 & 46 \\
\hline Number of spermatozoa/ejaculate $\left(\times 10^{9}\right)$ & 1.9 & 1.8 & 1.3 & 0.004 & 8.9 \\
\hline Individual motility (\%) & 48.9 & 29.9 & 57.5 & 0 & 90 \\
\hline Normal spermatozoa (\%) & 70.8 & 22.5 & 78.7 & 2.6 & 98.5 \\
\hline \multirow[t]{2}{*}{ Clinical score } & \multicolumn{5}{|c|}{ Seminal score } \\
\hline & Poor & Fair & Good & Total & \\
\hline Poor & 23 & 21 & 12 & 56 & \\
\hline Fair & 31 & 36 & 38 & 105 & \\
\hline Good & 12 & 14 & 31 & 57 & \\
\hline Total & 66 & 71 & 81 & 218 & \\
\hline
\end{tabular}

*BCS : body condition score; **SC : scrotal circumference 
Table 4 Distribution of the results (number of rams) and concordance of serological tests

\begin{tabular}{|c|c|c|c|c|c|}
\hline \multirow[t]{2}{*}{$\mathrm{CFT}^{*}$} & \multicolumn{5}{|c|}{ I-ELISA (30-60 \%)** } \\
\hline & Negative & Doubtful & Positive & Total & \\
\hline Negative & 95 & 9 & 32 & 136 & \\
\hline Positive & - & 1 & 80 & 81 & \\
\hline Total & 95 & 10 & 112 & 217 & \\
\hline \multirow[t]{2}{*}{ CFT } & \multicolumn{5}{|c|}{ I-ELISA $(45 \%)^{* *}$} \\
\hline & Negative & Positive & Total & & \\
\hline Negative & 100 & 36 & 136 & & $K=0.676$ \\
\hline Positive & - & 81 & 81 & & $95 \%$ Cl [0.584;0.767] \\
\hline Total & 100 & 117 & 217 & & \\
\hline
\end{tabular}

*One anti-complementary serum not included in the analysis **Cut-off: $30-60 \%$ or $45 \%$

K: Cohen's kappa coefficient; $95 \% \mathrm{Cl}=95 \%$ confidence interval

\section{Serological results}

Detailed serological results are given in Table 4. As expected from the inclusion criteria, about half of animals were effectively seropositive but more clearly in I-ELISA (53.7 \%) when a unique cut-off of $45 \%$ was chosen according to Praud et al. [8] than in CFT (37.2\%).

One serum presented an anti-complementary activity and was removed from the analysis. The two tests gave concordant results for 181 animals (83.0 \%) with a higher number of positive results with I-ELISA than with CFT (117 vs. 81). The agreement between CFT and I-ELISA (45\%), evaluated by the Cohen's kappa coefficient, was good: $\mathrm{K}=0.676$ (95 \% CI [0.584; 0.767]).

\section{Relationship between serological results and clinical alterations}

There was a significant relationship between positive serological results and clinical alterations $(p<0.001)$. About $64 \%$ and $81 \%$ of animals with clinical alterations of the genital tract (see above) were positive for CFT and ELISA respectively (Table 5 ) but only $58 \%$ of animals positive to CFT (47/81) and $50 \%$ of animals positive to I-ELISA (59/117) presented clinical alterations of the genital tract.

\section{Relationship between serological results and seminal score}

Most of I-ELISA-positive animals (83 \%) presented a poor or fair seminal score and $40 \%$ of I-ELISA-negative rams presented such a poor or fair seminal score as well (Tables 6 and 7). The Fisher exact test showed a significant relationship between the positive serological status of rams and the low seminal score $(p<0.001)$.

\section{Bacteriological results}

Only 198 results were interpretable; for 16 samples, cultures were contaminated by overgrowing microorganisms that made the interpretation impossible; and 4 samples were not tested due to an unsufficient quantity of semen. B. ovis was isolated in the semen of 89 rams, i.e. $44.95 \%$ of 198 sampled animals with culture results.

There was a significant relationship $(p<0.05)$ between culture results and clinical score (Table 6). Among the parameters determining the clinical score, only BCS $(p<$ $0.01)$ and lesions $(p<0.001)$ influenced significantly the bacteria shedding.

There was a significant relationship $(p<0.001)$ between culture results and seminal score (Table 8 ).

A strong relationship between culture and CFT positive results $(p<0.001)$ as well as I-ELISA positive results $(p<0.01)$ was also evidenced: almost all rams shedding $B$. ovis in their semen gave I-ELISA positive results (Table 9). The agreement between culture and CFT was almost $\operatorname{good}(\mathrm{K}=0.597 ; 95 \% \mathrm{CI}[0.484 ; 0.709]$ and good between culture and I-ELISA (45\%) (K=0.710; $95 \% \mathrm{CI}$ [0.615;0.806]).

\section{Discussion}

Our study clearly demonstrates that the B. ovis infection induces genital lesions and alters the semen quality, leading to an alteration of sexual function of the rams. However this study performed in field conditions on 218 rams included a bias associated to the selection of flocks (voluntary instead of randomly selected) and rams (selected by their owners instead of random), leading to a study sample that could be not representative of the ram population in South-Eastern France.

Table 5 Relationship between serological tests and clinical (testicular and/or epididymal) alterations

\begin{tabular}{|c|c|c|c|c|c|c|}
\hline \multirow{3}{*}{$\begin{array}{l}\text { Clinical } \\
\text { alterations }\end{array}$} & \multicolumn{6}{|c|}{ Serological results } \\
\hline & \multicolumn{3}{|l|}{$\mathrm{CFT}^{*}$} & \multicolumn{3}{|c|}{ I-ELISA $(45 \%)^{* *}$} \\
\hline & Negative & Positive & & Negative & Positive & \\
\hline Yes & 26 & 47 & $p<0.001^{* * *}$ & 14 & 59 & $p<0.001^{* * *}$ \\
\hline No & 110 & 34 & & 87 & 58 & \\
\hline Total & 136 & 81 & & 101 & 117 & \\
\hline
\end{tabular}

*One anti-complementary serum not included in the analysis

${ }^{* *}$ Cut-off: $45 \%$

*** Fisher exact test 
Table 6 Pathological changes in spermatozoa

\begin{tabular}{llll}
\hline Pathological changes of spermatozoa & Mean \pm SD* $^{*}$ & Median* & Maximum* \\
\hline Detached head & $10.8 \pm 14.6$ & 4.1 & 0.7 \\
Elongated head & $0.1 \pm 0.4$ & 0 & 4.0 \\
Proximal droplet & $0.4 \pm 1.6$ & 0 & 19.5 \\
Distal droplet & $0.1 \pm 0.6$ & 1.6 & 6.1 \\
Isolated tail & $5.2 \pm 7.3$ & 5.6 & 38.5 \\
Bent tail & $7.6 \pm 7.2$ & 2.0 & 51.5 \\
Coiled tail & $3.8 \pm 4.8$ & 0.5 & 28.5 \\
Broken tail & $1.2 \pm 1.5$ & 8.0 \\
\hline
\end{tabular}

*\%

Another bias is due to the staining kit that probably induced a high percentage of artifactual sperm abnormalities, mainly reflex midpieces, as reported by Kimberling and Parsons [9]. As a consequence, we did not take into account this anomaly for the calculation of the percentage of normal spermatozoa.

The point-score system that we used to classify the rams was adapted from Ley et al. [7]. The clinical and seminal criteria and/or the thresholds selected were close, but slightly different, from those of Ley et al. [7], Kimberling and Parsons [9] and Van Metre et al. [10].

In rams originated from $B$. ovis infected flocks, only $26 \%$ and $37 \%$ of rams had good clinical or seminal score respectively. This result, lower than the $70 \%$ of rams classified as questionable or unsatisfactory breeders in previous studies $[7,10]$, can be explained by the high prevalence of $B$. ovis infection in our rams population. Furthermore, the low rate of good clinical and seminal scores in the studied population might be due not only to $B$. ovis infection but also to other infectious or noninfectious causes of orchi-epididymitis, as reported previously [2]. A significant relationship was observed between clinical and seminal scores, but this was primarily explained by clinical alterations, not by differences in SC and age. Indeed, SC may be related to

Table 7 Relationship between I-ELISA results and seminal score

\begin{tabular}{llllll}
\hline & & \multicolumn{5}{c}{ Seminal score } & \\
& & Poor & Fair & Good & Total \\
\hline I-ELISA (30-60 \%) & Negative & 6 & 32 & 57 & 95 \\
& Doubtful & & 5 & 6 & 11 \\
& Positive & 60 & 34 & 18 & 112 \\
& Total & 66 & 71 & 81 & 218 \\
I-ELISA (45 \%) & Negative & 6 & 34 & 61 & 101 \\
& Positive & 60 & 37 & 20 & 117 \\
& Total & 66 & 71 & 81 & 218 \\
\hline
\end{tabular}

genital abnormalities but also to several factors, such as age, breed, season, testicular tone, nutrition, parasite and other concurrent disease status [11].

The comparison of the serological results obtained by the different methods (CFT and I-ELISA) confirms the higher sensibility of the I-ELISA compared to CFT, already reported by Vigliocco et al. [12], Praud et al. [8] and Ridler et al. [13]; moreover, I-ELISA can detect infected rams earlier than CFT [11].

In a recent study, Ridler et al. [13] considered that a "suspicious" result is a disadvantage of the B. ovis IELISA and concluded that rams presenting such a result should be isolated and re-tested 2 to 4 weeks later.

Previously, Praud et al. [8] considered that the use of one or two thresholds can depend on the strategy of control of the disease. In an a priori healthy area, a high threshold limits the risk of "false positive" results but, at the opposite, in an infected area, a low threshold increases the diagnostic sensitivity; thus, according to the situations and strategic choices, the class of "doubtful" animals can be grouped with that of "healthy" or "infected". Under the conditions of our study, I-ELISA-45, with a unique threshold, as proposed by Praud et al. [6], was more sensitive than CFT to detect rams carrying lesions and producing semen of poor quality.

Almost all rams shedding $B$. ovis in their semen were seropositive (mainly in I-ELISA) but approximately $25 \%$ of the seropositive rams were culture-negative for $B$. ovis. This can be due to the intermittent excretion of $B$. ovis, as previously reported [3] and, also, to the fact that the rams were sampled only once $[1,13]$.

According to previous reports [4, 13, 14], we observed a significant relationship between culture-positive results (i.e. excretion of B. ovis in semen) and genital alterations or poor semen quality. Although significantly linked, seropositivity and genital tract alterations were not systematically associated, as previously described by Blasco [3], Ridler and West [15] and Van Metre et al. [10]. Moreover, approximately one third of B. ovis-shedding 
Table 8 Relationship between bacteriology and clinical or seminal score

\begin{tabular}{|c|c|c|c|c|c|c|c|c|c|c|}
\hline & & & \multicolumn{4}{|c|}{ Clinical score $(n=218)$} & \multicolumn{4}{|c|}{ Seminal score $(n=218)$} \\
\hline & & & Poor & Fair & Good & & Poor & Fair & Good & \\
\hline \multirow[t]{6}{*}{ B. ovis culture results $(n=198)$} & Negative & No. & 22 & 54 & 33 & $p<0.05^{* *}$ & 17 & 33 & 59 & $p<0.01^{* *}$ \\
\hline & & (\%) & $(10.1)$ & $(24.8)$ & $(15.1)$ & & $(7.8)$ & $(15.1)$ & $(27.1)$ & \\
\hline & Positive & No. & 29 & 42 & 18 & & 43 & 34 & 12 & \\
\hline & & (\%) & (13.3) & (19.3) & $(8.3)$ & & $(19.7)$ & $(15.6)$ & $(5.5)$ & \\
\hline & Contaminated or not tested* & No. & 5 & 9 & 6 & & 6 & 4 & 10 & \\
\hline & $(n=20)$ & (\%) & $(2.3)$ & $(4.1)$ & $(2.8)$ & & $(2.8)$ & $(1.8)$ & $(4.6)$ & \\
\hline
\end{tabular}

*16 semen culture contaminated, 4 samples not tested

**Fisher exact test

rams presented clinical alterations [15], suggesting that $B$. ovis isolation in semen could be more precocious than the detection of epididymitis, as reported by Ridler et al. [13]. Furthermore, clinical alterations may be undetectable in the presence of active excretion of B. ovis and serological positive results, especially in chronically infected rams, as described previously by Worthington et al. [16]. In this study, 73 rams showed detectable lesions but 89 gave culture positive results while 81 and 117 gave positive results in CFT and I-ELISA respectively.

\section{Conclusion}

This study performed in B. ovis infected flocks of the "PACA" region, confirmed the findings of various previous field or experimental studies. As regards eradication of $B$. ovis infection, the following recommendations could be proposed: in areas with a low-to-medium prevalence, the eradication of $\mathrm{CE}$ is possible by using a test-and-slaughter approach, incorporating compounding tests to increase the overall testing sensitivity and reduce the likelihood of false negatives, as proposed by Blasco [3], Ridler and West [15] Praud et al. [8] and Ridler et al. [13]. Such an approach might include a combination of serological (I-ELISA and CFT) and auxiliary tests (genital palpation and, when possible, semen

Table 9 Relationship between semen bacteriology and serology

\begin{tabular}{ccccccc}
\hline \multirow{2}{*}{$\begin{array}{l}\text { Serological } \\
\text { results }\end{array}$} & \multicolumn{5}{c}{ B. ovis culture results } \\
& Negative & Positive & \\
\hline CFT & Nogative & 96 & $(48.7)$ & 25 & $(12.7)$ & $p<0.001^{*}$ \\
$(n=197)$ & Positive & 13 & $(6.6)$ & 63 & $(32.0)$ & $\mathrm{K}=0.597^{* *}$ \\
I-ELISA (45 \%) & Negative & 84 & $(42.4)$ & 4 & $(2.0)$ & $p<0.001 ; \mathrm{K}=0.710$ \\
$(n=198)$ & Positive & 25 & $(12.6)$ & 85 & $(42.9)$ & \\
\hline
\end{tabular}

*Fisher exact test

**K: Cohen's kappa coefficient culture). This approach, which must be applied before each breeding season as well as before any ram introduction in free flocks, might allow for more rapid identification and removal of chronic shedders. In areas with a high prevalence, this strategy might be economically unsustainable. Despite recently developed B. ovis mutant attenuated strains gave promising protecting results in mice $[17,18]$, the $B$. melitensis Rev.1 vaccine remains up to now the only vaccine with proven efficacy against $B$. ovis infection in rams [2]; therefore, Rev.1 vaccination would be certainly the most economical and practicable tool for midterm control in such situations [3], in particular in areas where export of rams or ram semen is not an economical priority and where the official brucellosis-free status is not a short-term goal.

\section{Abbreviations}

B. ovis: Brucella ovis; PACA: Provence-Alpes-Côte d'Azur; BCS: Body condition score; SC: Scrotal circumference; CE: Contagious epididymitis; CFT: Complement fixation test; I-ELISA: Indirect enzyme-linked immunosorbent assay.

\section{Competing interests}

The authors declare that they have no competing interests.

\section{Authors' contributions}

$\mathrm{NPH}, J L C, L E, M M, M P, A S$ and $C T$ performed clinical examination of rams, semen collection and blood sampling for serology. XB, AS and CT performed semen evaluation. BGB directed the laboratory test performance. XB, NPH, FL and MP performed data analyses. NPH, XB and BGB drafted the manuscript. $\mathrm{NPH}, \mathrm{XB}, J \mathrm{LC}, \mathrm{MM}, \mathrm{MP}$ and $\mathrm{BGB}$ contributed to the conception and design of the whole study and revised the manuscript. All the authors approved the final manuscript.

\section{Authors' information}

$\mathrm{NPH}$ and XB: DVM, PhD, professors of Theriogenology, National Veterinary School of Toulouse.

JLC and LE: DVM, Veterinary practitioners, Veterinary Advisors of the Groupement de Défense Sanitaire des Alpes de Haute Provence and des Bouches du Rhône respectively.

MP: MSc, technical manager, Fédération Régionale des Groupements de Défense Sanitaire Provence Alpes Côte d'Azur

BGB: DVM, PhD, Senior Research Director, Head of the EU/OIE/FAO

Brucellosis Reference Laboratory (ANSES).

FL: PhD, assistant professor of Biostatistics, National Veterinary School of Toulouse.

AS and CT: graduating students (actually DVM), National Veterinary School of Toulouse. 


\section{Acknowledgements}

We thank the stock-breeders, PROVALP, Nicolas CORBOZ and the staff of the FRGDS-PACA for their active participation to this study. Moulay-Ali CHERFA, Yannick CORDE, Antoine DRAPEAU and Gilles LE CARROU (Anses) are also duly acknowledged for their expert technical assistance.

\section{Author details}

'Université de Toulouse, INP-ENVT, UMR 1331-Toxalim, F-31076 Toulouse, France. ${ }^{2}$ Université de Toulouse, INP-ENVT, UMR 1225-IHAP and UMT Maîtrise de la santé des troupeaux de petits ruminants, F-31076 Toulouse, France. ${ }^{3}$ Groupement de Défense Sanitaire des Alpes de Haute Provence, F-04000 Digne les Bains, France. ${ }^{4}$ Groupement de Défense Sanitaire des Bouches du Rhône, F-13626 Aix en Provence, France. ${ }^{5}$ Université de Toulouse, INP-ENVT, Unité de Biostatistiques, F-31076 Toulouse, France. ${ }^{6}$ Fédération Régionale des Groupements de Défense Sanitaire Provence Alpes Côte d'Azur, F-04100 Manosque, France. ${ }^{7}$ Paris-Est University/French Agency for Food, Environmental and Occupational Health and Safety (ANSES), EU/OIE/FAO Brucellosis Reference Laboratory, 94701 Maisons-Alfort, France. ${ }^{8}$ Present Address: ANSES, European \& International Affairs Department, 14 rue Pierre et Marie Curie, F-94701 Maisons-Alfort, Cedex, France.

Received: 8 January 2015 Accepted: 18 May 2015

Published online: 30 May 2015

\section{References}

1. Ficapal A, Jordana J, Blasco JM, Moriyón I. Diagnosis and epidemiology of Brucella ovis infection in rams. Small Ruminant Res. 1998;29:13-9.

2. OIE (World Organisation for Animal Health). Ovine epididymitis (Brucella ovis). In: OIE, editor. Manual of Diagnostic Tests and Vaccines for Terrestrial Animals. Paris: OlE; 2014. [http://www.oie.int/fileadmin/Home/eng/ Health standards/tahm/2.07.09 OVINE EPID.pdf].

3. Blasco JM. Brucella ovis. In: Nielsen K, Duncan JR, editors. Animal Brucellosis. Boca Raton: CRC Press; 1990. p. 351-78.

4. Carvalho Junior CA, Moustacas VS, Xavier MN, Costa EA, Costa LF, Silva TMA, et al. Andrological, pathologic, morphometric, and ultrasonographic findings in rams experimentally infected with Brucella ovis. Small Ruminant Res. 2012;102:213-22.

5. Russell A. Body condition scoring in sheep. In Pract. 1984;6:91-3

6. Kenyon PR, Maloney SK, Blache D. Review of sheep body condition score in relation to production characteristics. New Zeal J Agr Res. 2014;57:38-64.

7. Ley WB, Sprecher DJ, Thatcher CD, Pelzer KD, Umberger SH. Use of the point-score system for breeding soundness examination in yearling Dorset, Hampshire and Suffolk rams. Theriogenology. 1990;34:721-33.

8. Praud A, Champion JL, Corde Y, Drapeau A, Meyer L, Garin-Bastuji B. Assessment of the diagnosis sensitivity and specificity of an indirect I-ELISA kit for the diagnosis of Brucella ovis infection in rams. BMC Vet Res. 2012;8:68.

9. Kimberling CV, Parsons GA. Breeding soundness evaluation and surgical sterilization of the ram. In: Youngquist RS, Threlfall WR, editors. Current therapy in large animal theriogenology. 2nd ed. St Louis: Saunders Elsevier; 2007. p. 620-8.

10. Van Metre DC, Sangeeta Rao S, Kimberling CV, Morley PS. Factors associated with failure in breeding soundness examination of Western USA rams. Prev Vet Med. 2012;105:118-26.

11. Ridler AL, Smith SL, West DM. Ram and buck management. Anim Reprod Sci. 2012;130:180-3.

12. Vigliocco AM, Silva Paulo PS, Mestre J, Briones GC, Draghi G, Tossi M, et al. Development and validation of an indirect enzyme immunoassay for detection of ovine antibody to Brucella ovis. Vet Microbiol. 1997;54:357-68.

13. Ridler AL, Smith SL, West DM. Seroconversion and semen shedding in rams experimentally infected with Brucella ovis. New Zeal Vet J. 2014;62:47-50

14. Kott RW, Halver GC, Firehammer B, Thomas VM. Relationships between Brucella ovis semen culture and various semen and serology parameters. Theriogenology. 1988;29:961-70.

15. Ridler AL, West DM. Control of Brucella ovis Infection in Sheep. Vet Clin N Am-Food A. 2011;27:61-6.

16. Worthington R, Stevenson B, De Lisle G. Serology and semen culture for the diagnosis of Brucella ovis infection in chronically infected rams. New Zeal Vet J. 1985:33:84-6.
17. Sancho P, Tejedor C, Sidhu-Muňoz RS, Fernández-Lago L, Vizcaíno N. Evaluation in mice of Brucella ovis attenuated mutants for use as live vaccines against B. ovis infection. Vet Res. 2014;45:61.

18. Soler-Lloréns P, Gil-Ramírez Y, Zabalza-Baranguá A, Iriarte M, Conde-Álvarez R, Zúñiga-Ripa A, et al. Mutants in the lipopolysaccharide of Brucella ovis are attenuated and protect against $B$ ovis infection in mice. Vet Res. $2014 ; 45: 72$.

\section{Submit your next manuscript to BioMed Central and take full advantage of:}

- Convenient online submission

- Thorough peer review

- No space constraints or color figure charges

- Immediate publication on acceptance

- Inclusion in PubMed, CAS, Scopus and Google Scholar

- Research which is freely available for redistribution 